\title{
Cross-Validation of Paranoid- Depressive Scale and Functional MRI: New Paradigm for Neuroscience Informed Clinical Psychopathology
}

\author{
Drozdstoy Stoyanov ${ }^{1 *}$, Sevdalina Kandilarova ${ }^{1}$, Zlatoslav Arabadzhiev $^{1}$, \\ Rossitsa Paunova ${ }^{1}$, André Schmidt ${ }^{2}$ and Stefan Borgwardt ${ }^{2}$ \\ ${ }^{1}$ Neuropsychiatry and Brain Imaging Group, Department of Psychiatry and Medical Psychology, Plovdiv Medical \\ University, Plovdiv, Bulgaria, ${ }^{2}$ Neuropsychiatry and Brain Imaging Group, Department of Psychiatry, University of Basel, \\ Basel, Switzerland
}

OPEN ACCESS

Edited by:

Roumen Kirov,

Institute of Neurobiology (BAS),

Bulgaria

Reviewed by:

Sérgio Saraiva,

Centro Hospitalar Psiquiátrico

de Lisboa, Portugal

Massimiliano Aragona,

Società Italiana di Medicina

Migratoria, Italy

*Correspondence:

Drozdstoy Stoyanov

drozdstoy.stoyanov@mu-plovdiv.bg

Specialty section: This article was submitted to

Neuroimaging and Stimulation,

a section of the journal

Frontiers in Psychiatry

Received: 11 July 2019 Accepted: 05 September 2019 Published: 27 September 2019

Citation:

Stoyanov D, Kandilarova S,

Arabadzhiev Z, Paunova $R$,

Schmidt A and Borgwardt S (2019)

Cross-Validation of Paranoid-

Depressive Scale and Functional

MRI: New Paradigm for

Neuroscience Informed Clinical

Psychopathology.

Front. Psychiatry 10:711.

doi: 10.3389/fpsyt.2019.00711
There is reported a study performed with a novel paradigm aiming at investigation of the translational validity of von Zerssen's paranoid-depression scale and its fMRI correlates in terms of focus on exploration of the results on the contrast between the Paranoid Specific (DP) blocks and the Depression Specific (DS) blocks. Patients with schizophrenia demonstrated significant activations in a number of regions (right angular gyrus, left posterior cingulate and precuneus, right transverse temporal gyrus) during responses to paranoia versus depression items which differ topologically from those found in patients with major depression (left middle cingulate and right superior temporal gyrus). The direct comparison between the groups, however, did not yield any residual activations after correction.

Keywords: functional MRI, depression, schizophrenia, validation, psychopathology

\section{INTRODUCTION}

Considering the status of psychiatry as a hybrid discipline which embraces both the natural sciences and the humanities $(1,2)$, we attempt to deliver a novel, experimentally fostered concept of translational validity, which is a non-conventional and instrumentalist approach to validation (3).

As discussed in earlier publications (4-6) clinical and neurobiological measures are considered valid for different reasons inside their own divergent domains. All disciplines concerned with mental health establish internal or intra-correlative validity, i.e. psychological scales are typically validated against other psychological measures, and neurobiological measures are validated with other neurobiological tests. Psychiatric assessment tools represent a circle of validation between first-person measures (self-evaluation inventories) and third-person perspective, the psychiatric interviews (7). What is still missing is the inter-correlative or inter-disciplinary validity which entails consistent inter-domain translation. In practical terms the lack of consistent inter-domain translation is undermining the validity of psychiatric classifications as well as the implementation of the research findings in clinical practice (8).

Furthermore, we consider of critical importance the notion of "state dependence" as contrasted with the traditional "state independence" of biomarkers (9). State dependence means in this context that certain correlations are directly relevant and may be specific to the current mental state, yet 
not necessarily to the diagnosis in the medical sense. This is why the clinical and biological measures are beeing performed simultaneously in our paradigm (10).

The translation takes place on two levels according to the established psychometric vallidation standards: convergent and divergent (11). In first place the corresponding empirical measures are cross-validated on convergent level, e.g. depression clinical rating scale and blood oxygenation level dependent (BOLD) activation levels from functional magnetic resonance imaging (fMRI) during processing of neutral items in patient versus healthy control population (12). After that the construct is cross-validated in the same manner against another, presumably divergent clinical construct, e.g. paranoia. However discriminative power is not expected to be strong enough to underpin robust discrimination across nosological entities, but rather patterns of activation that may underpin the different psychopathological constructs and relevant measures.

In our previous studies we have tested the convergent validity by employing an fMRI paradigm using two types of visual stimuli-diagnostically specific (DS) - representing items from von Zerssen's depression subscale (13) and diagnostically neutral (DN) - from an interest scale. Thus, we have been able to demonstrated that in healthy controls, contrasting the two types of stimuli (DS vs DN) yielded no significant brain activations, and the correlation analyses did not find a relationship between brain activations and the total score of the DS statements. On the other hand, in depressed patients contrasting the DS to the $\mathrm{DN}$ stimuli produced significant activations in several brain regions, and there were positive correlations with the DS score in several activation clusters $(12,14)$. In this manner, we were able to confirm the sensitivity of the method (its ability to distinguish healthy controls from depressed patients), still we had to address its specificity (different patterns of brain activation behind different clinical constructs and respective measures). As it has already been stated, such patterns are not likely to trascend to the level of nosological specificity.

To handle this last issue and to test the divergent validity, we further developed our paradigm with the specific aim of investigating the translational validity of von Zerssen's paranoia-depression scale (13) and its fMRI correlates during their simultaneous implementation in patients with depression and schizophrenia.

\section{METHODS}

\section{Subjects}

We recruited 35 psychiatric patients with either a diagnosis of schizophrenia ( $n=15$, mean age $37.1 \pm 12$ years, 11 males), or depressive episode ( $n=20$, mean age $42.3 \pm 12.1$ years, 5 males $)$ in the context of major depressive disorder $(n=10$, mean age $39.9 \pm$ 11.9 years, 4 males) or bipolar disorder $(n=10$, mean age $44.8 \pm$ 12.5 years, 2 males). Subjects were assessed by an experienced psychiatrist (ZA) using the general clinical interview and the structured Mini International Neuropsychiatric Interview (M.I.N.I 6.0) (15) as well as the Montgomery-Åsberg Depression
Rating Scale (MADRS) (16) and the Positive and Negative Syndrome Scale (PANSS) (17).

Patients were excluded if they had a comorbid psychiatric disorder (such as anxiety, substance related disorder), major medical illness, neurological disease, history of head trauma with loss of consciousness, or metal implants not compatible with the MRI. All participants provided a written informed consent complying with the Declaration of Helsinki and the study was approved by the University's Ethics Committee.

\section{MR Scanning}

The scanning of the participants was performed on a 3T MRI system (GE Discovery 750w). The MR protocol included high resolution structural scan (Sag 3D T1 FSPGR sequence), with slice thickness $1 \mathrm{~mm}$, matrix $256 \times 256$, TR (relaxation time) 7.2 ms, TE (echo time) 2.3 , and flip angle $12^{\circ}$, and a functional scan (2D EPI sequence), with slice thickness $3 \mathrm{~mm}$, matrix $64 \times 64$, TR $2000 \mathrm{msec}$, TE $30 \mathrm{msec}$, and flip angle $90^{\circ}$. Before each functional scan 5 dummy time series were acquired.

\section{fMRI Stimuli and Procedure}

We used a standard block-design with three different active conditions and one rest condition, with a total duration of $11 \mathrm{~min}$ and $44 \mathrm{~s}$. Each active block lasted for $32 \mathrm{~s}$ and consisted of four text statements presented for $8 \mathrm{~s}$ each using NordicNeuroLab VisualSystem. For the Depression Specific (DS) blocks the statements were taken from the von Zerssen depression subscale ("I cry easily," "I am more sensitive to criticism than I was before"), while for the Paranoia Specific (DP) blocks they were taken from the paranoid subscale ("Other people constantly follow and control me"). As in our previous study (12), there were also Diagnostically Neutral (DN) blocks consisting of four statements from a questionnaire about general interests and likes (such as "I like to write books or plays," "I like to repair household appliances," etc.). Under each written statement four possible answers ("completely true," "mostly true," "somewhat true," "not true") and the respective four response buttons (upper left, lower left, lower right, upper right) were presented. There were four blocks of each type, alternating between the three active conditions (DS, DN, and DP) followed by a $20 \mathrm{~s}$ resting block with a fixation cross in the middle of the screen (DS DN_DP_D). For the active conditions, the participants were instructed to read the statements carefully and to respond with a button press according to their level of agreement, and for the passive condition, to focus on the fixation cross without thinking of anything in particular.

\section{fMRI Data Analysis}

Data were analyzed using the SPM 12 (Statistical Paramertic Mapping, http://www.fil.ion.ucl.ac.uk/spm/) software running on MATLAB R2015 for Windows. The preprocessing included the following steps: i) realignment of the functional data for correction of head motion, ii) co-registration between the highresolution anatomical image and the functional scans, iii) intraindividual estimation of spatial registration parameters based on the anatomical image, and iv) transformation of the co-registered functional data to standardized MNI (Montreal Neurological 
Institute) space, followed by v) spatial smoothing with a $8 \mathrm{~mm}$ full-width-at-half-maximum Gaussian kernel.

First-level analysis was conducted using a general linear model (GLM) applied to the time series, convolved with a canonical hemodynamic response function. Nuisance covariates included the six rigid body motion parameters. T-contrasts were defined for the active vs passive conditions. The resulting individual contrast maps from each comparison were then used in a second-level random-effects analysis to test for differences between the two patient groups (schizophrenia > depression and depression $>$ schizophrenia). Furthermore, ANOVA design was used to explore the three clinical diagnosis-schizophrenia, major depression, and bipolar depression. The level of significance was set to $\mathrm{p}>0.05$ FWE (Family Wise Error) corrected using an uncorrected cluster-forming threshold of $\mathrm{p}<0.001$, and gender was used as a covariate in all second-level analysis.

Following the logic of our study in terms of differentiating between the clinical diagnosis of schizophrenia and depression by means of simultaneous application of fMRI and a clinical assessment tool (e.g. von Zerssen paranoia-depression scale), we focused our exploration of the results on the contrast between the Paranoia Specific (DP) blocks and the Depression Specific (DS) blocks.

\section{RESULTS}

\section{Demographic and Clinical Characteristics}

The two patient groups did not differ significantly in their demographic and clinical characteristics (such as age, education, illness duration) except for the sex distribution, which was complying with the clinical reality, e.g. male prevalence in the schizophrenia group and the opposite for the depression group (Table 1).

The two depression subsamples-unipolar and bipolar-were not significantly different in their demographics as well as in their clinical features.

\section{Comparative Analysis Across Schizophrenia and Depression Patients}

The direct comparison of the DP > DS contrast between the two clinical populations produced multiple clusters of activation with significance level $<0.001$ which did not survive above the 0.05 p-level after FWE correction. On the same inter-group level and prior to inclusion of gender as co-variate there was localized a cluster with sigin the right angular gyrus, with peak activation significance level $\mathrm{p}=0.036$, consistent with our findings on group level as described bellow. This cluster was above the level of significance after inclusion of gender as co-variate, which demonstrates the critical role of gender confound in such study design (discussed as limitation). On the group level (one sample $t$-test) the schizophrenic patients demonstrated residual activations in several clusters encompassing medial parietal and limbic structures (posterior cingulum and precuneus), as well as temporal and subcortical regions (for details see Table 2). The depressed patients, on the other hand, showed only two clusters with peak activations in middle cingulate and in superior temporal gyrus (Table 2). An illustration of these results is given in Figure 1.

\section{Comparative Analysis Across Schizophrenia, Major Depression, and Bipolar Disorder}

In addition, a one-way ANOVA model of the DP > DS contrast differentiated between the two depressed patients groups (unipolar and bipolar) and the schizophrenic group. Significant difference was found only between schizophrenic subjects and those with major depression in a single cluster located to the right pre/postcentral gyrus (201 voxels, $\mathrm{p}=0.05 \mathrm{FWE}$ ) that is more activated in schizophrenia. The other betweengroup comparisons did not reach statistical significance. On the intragroup level, the schizophrenic subjects demonstrated residual activations almost identical to the ones revealed by the two-sample $t$-test, while the bipolar patients had only one cluster of greater activation located to the left middle cingulate gyrus (201 voxels, $\mathrm{p}=0.012 \mathrm{FWE}$ ). No residual activations were found in major depression.

\section{DISCUSSION}

The main finding of our study maight be summarized as follows: patients with schizophrenia demonstrated significant activations in a number of regions (right angular gyrus, left posterior cingulate and precuneus, right transverse temporal gyrus) during responses to paranoia versus depression items (DP > DS contrast) which differ topologically from those found in patients with major depression (left middle cingulate and right superior temporal

TABLE 1 | Demographic and clinical characteristics of all participants.

Schizophrenia patients $(n=15)$

Episode duration (weeks)
Age $($ mean $\pm \mathrm{SD})$

$\operatorname{Sex}(\mathrm{M} / \mathrm{F})$

Education (secondary/higher)

Age at onset (years)

Illness duration (months)

$37.1 \pm 12$
$11 / 4$
$10 / 5$
$27.7 \pm 8.2$
$110 \pm 95$
$9.1 \pm 7.1$

SD, Standard Deviation. alndependent samples $t$-test, ${ }^{b} \chi^{2}$-test, ${ }^{*} p<0.05$
Depressed patients $(n=20)$

Statistical

significance

$42.3 \pm 12.1$

$5 / 15$

$11 / 9$

$32.1 \pm 10.9$

$140 \pm 93$

$15.3 \pm 10$ $0.210^{a}$

*0.005 ${ }^{\mathrm{b}}$

$0.486^{b}$

$0.267^{\mathrm{a}}$

$0.406^{a}$

$0.307^{a}$ 
TABLE 2 | Clusters of significantly greater activations in schizophrenic and depressed patients when answering to psychosis items compared to depression items (DP > DS contrast)

\begin{tabular}{|c|c|c|c|c|c|}
\hline \multirow[t]{2}{*}{ Anatomical localization } & \multirow{2}{*}{$\begin{array}{l}\text { Cluster size } \\
\text { (voxels) }\end{array}$} & \multicolumn{3}{|c|}{ Peak MNI coordinates } & \multirow{2}{*}{$\begin{array}{l}\text { p-value } \\
\text { (FWE) }\end{array}$} \\
\hline & & $\mathbf{x}$ & $\mathbf{y}$ & $\mathbf{z}$ & \\
\hline \multicolumn{6}{|l|}{ Schizophrenic patients } \\
\hline Right angular gyrus, SMG & 128 & 28 & -46 & 36 & 0.004 \\
\hline $\begin{array}{l}\text { Left posterior cingulus } \\
\text { and precuneus }\end{array}$ & 575 & -6 & -30 & 28 & 0.02 \\
\hline $\begin{array}{l}\text { Right transverse temporal } \\
\text { gyrus and anterior insula }\end{array}$ & 3756 & 28 & 8 & -10 & 0.05 \\
\hline Right caudate, thalamus & 76 & 20 & -12 & 22 & 0.05 \\
\hline \multicolumn{6}{|l|}{ Depressed patients } \\
\hline Left middle cingulate gyrus & 212 & -8 & -16 & 48 & 0.007 \\
\hline Right superior temporal gyrus & 1243 & 42 & -42 & 20 & 0.02 \\
\hline
\end{tabular}

gyrus). The direct comparison between the groups, however, did not yield any residual activations after correction. The significance of these findings will be discussed in the following lines.

One of the clusters of activations, produced by the DP > DS contrast in schizophrenia, was in the area of the right angular gyrus, which belongs to the inferior parietal lobule system. It has been reported to be involved in semantic processing, social cognition, and reasoning as a cross-modal hub to converge multisensory information (18). Reversed assymetry in this region has been associated with schzophrenia (19) where abberant modulation/ activation of the right angular gyrus was found as well (20).

Another cluster of residual activations in schizophrenic patients was stretching across the left posterior cingulate and precuneus which is implicated in autobiographical memory processing (21). It is assumed to contribute to episodic memory dysfunctions and abnormal functional connectivity that was found in schizophrenia $(22,23)$.

Amongst the significant clusters in our study, one was located in the right transverse temporal gyrus, or Heschl's convolution and anterior insula. The function of those regions is related to accoustic processing as an Inner voice, or internal subjective dialogue with oneself (24), as well as task-level control of focal attention (25), which are often disturbed in schizophrenia.

On the other hand, the significant activations in the group of depressed patients were located in left middle cingulate gyrus as well as right superior temporal gyrus which makes sense in the context of the functional role of those regions in depression $(26,27)$.

Our ANOVA findings are consistent with previously reported results (12) and may be explained with the activation of the motor cortex as patients with schzophrenia used more often their left hand to provide positive responses to paranoid items.

The limited inter-group contrast in our findings (not reaching statistical significance) might be explained by the discrepancy of psychometric or psychodiagnostic versus diagnostic i.e. nosological validity. Psychometric validity essentally covers validation of particular construct(s) by use of another method (here, translational validation of von Zerssen paranoia-depression scale with fMRI), and it appears to have been achieved in our model. However the nosological validity assumes the possibility to validate entire medical-psychiatric-diagnostic entity and it remains out of reach.
The items that compose diagnostic scales, however precise those may be in order to measure certain phenomenon, could create preconditions for terminological inaccuracy. The diagnostic validity of a psychological tool can trace out borders of a particular category, but this is not enough to make a diagnosis. Even formally precise psychometric tools as intelligence and cognitive assessment tests can be challenged when their results are viewed in a specific emotional and cultural context $(28,29)$.

Another possible explanation of the overlap between the activations related to the processing of paranoid and depressive items in both patient groups might lay in the clinical variations of depressive symptoms in affective disorders and schizophrenia. The background of rather "warm" affectivity, induced by melancholia and anxiety in the context of affective disorder and the "cold" affect in schizophrenic psychosis $(30,31)$, caused by blunted affect might be revealed on phenomenological level (during the clinical interview) but cannot be captured properly by brief clinical assessment tools widely employed in psychopathology.

In addition, negative or cognitive symptoms in schizophrenic patients may be mistakenly assessed by a psychological tool as depressive (pseudodepresive) and vice versa. Morover, about $25 \%$ to $50 \%$ of the patients with major depression have impairment of at least one cognitive sphere (32). The most common disturbances in cognitive functioning during a depressive episode are those of memory, attention, and the degree of processing of various incentive stimuli (33). This is to demonstrate that cognitive deficits can be seen as a central element in the course of a major depressive disorder, not just as secondary phenomena (31). In the same perspective, similar changes in cognitive functioning can be found in schizophrenic patients in the context of negative and cognitive symptoms, and it would be impossible for a psychological test to differentiate them on the level of nosological specificity.

To summarize it there are limitations concerning possible nosological specificity of evaluation measures in clinical psychiatry, as predicted in some earlier theoretical publications $(5,8)$.

\section{Limitations}

There are two major limitations which undermine generalizations from the current study. 


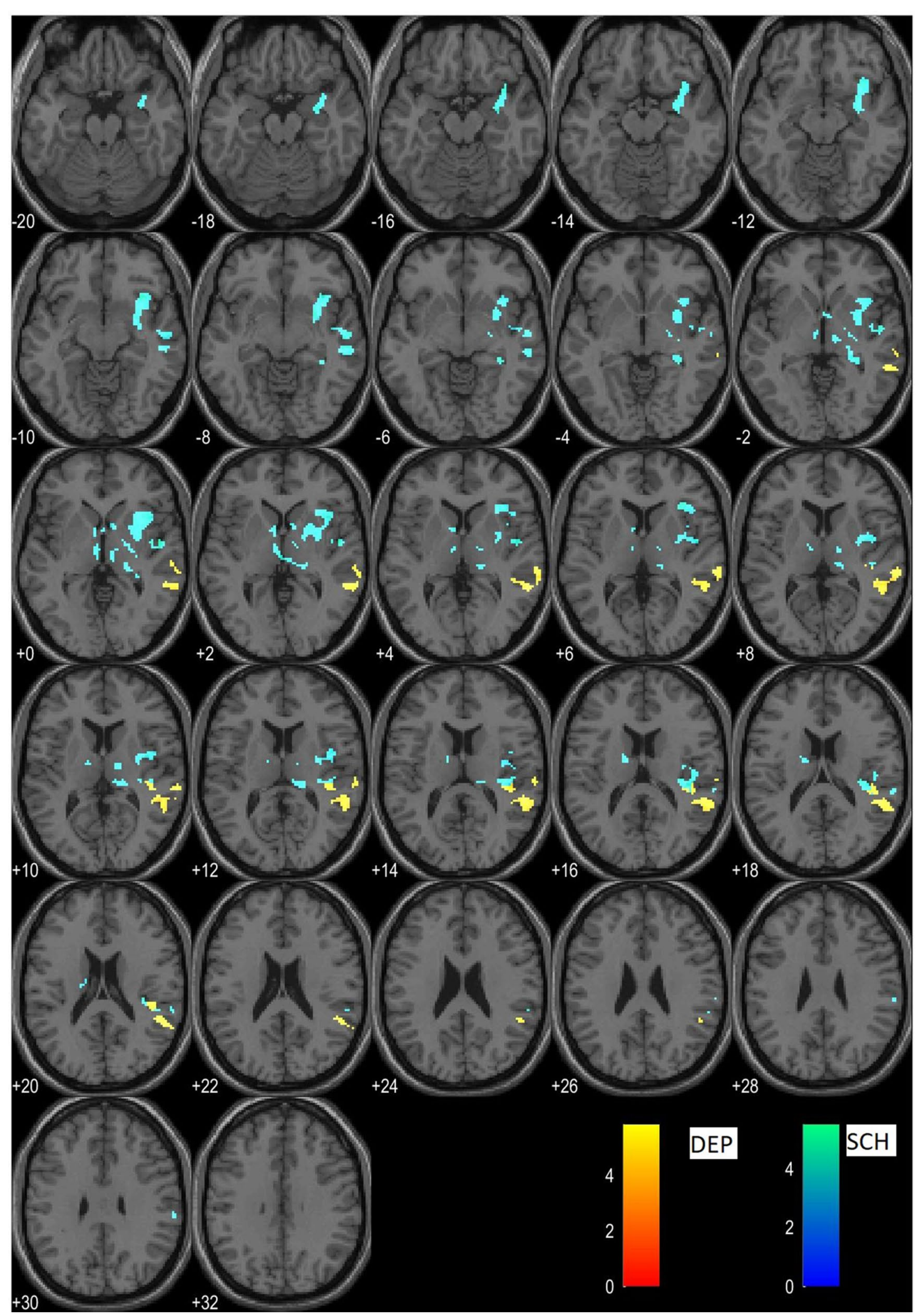

FIGURE 1 | Clusters of residual activations of the DP > DS contrast in schizophrenia (cyan) and in depression (yellow). 
The first is concerned with the small sample size, especially when the sample is subdivided into clinical diagnostic groups.

The second is the innovative and non-conventional approach to the the experimental paradigm design, which presents an issue for comparison with other studies in the field.

The third limitation apparently concerns the gender structure of the sample. In future replication studies gender balance will be of critical importance in order to bolster the significance of the results.

From a more general perspective those limitations might be addressed by expanding the research in translational neuroimaging using similar approach aiming to identify the functional MRI substrate behind clinical self-evaluation measures.

\section{DATA AVAILABILITY STATEMENT}

The datasets generated for this study are available on request to the corresponding author.

\section{REFERENCES}

1. Berrios GE. Psychiatry and its objects. Rev Psiquiatr Salud Ment (2011) 4(4):4. doi: 10.1016/j.rpsmen.2011.09.001

2. Markova I, Berrios GE. Neuroimaging in psychiatry:epistemological considerations. In: Zachar P, Stoyanov D, Aragona M, Jablenski A, editors. Alternative perspectives on psychiatric validation: DSM, ICD, RDoC, and beyond. Oxford University Press (2015). p. 112-27. doi: 10.1093/ med/9780199680733.003.0007

3. Stoyanov D, Borgwardt S, Varga S, Jablenski A. The problem of translational validity across neuroscience and psychiatry. In: Zachar P, Stoyanov D, Aragona M, editors. Alternative perspectives on psychiatric validation. Oxford University Press (2014). p. 128. doi: 10.1093/med/9780199680733.003.0008

4. Stoyanov D, Machamer PK, Schaffner KF, Rivera-Hernandez R. The challenge of psychiatric nosology and diagnosis. J Eval Clin Pract (2012) 18(3):704-9. doi: 10.1111/j.1365-2753.2012.01844.x

5. Stoyanov D, Machamer PK, Schaffner KF, Rivera-Hernández R. The metalanguage of psychiatry as cross-disciplinary effort: In response to Zachar. J Eval Clin Pract (2012) 18(3):710-20. doi: 10.1111/j.1365-2753.2012.01846.x

6. Stoyanov D, Stanghellini G, Broome M. Conceptual issues in psychiatric neuroimaging: an update. Curr Top Med Chem (2012) 12(21):2348-56. doi: $10.2174 / 156802612805289836$

7. Nordgaard J, Sass LA, Parnas J. The psychiatric interview: validity, structure, and subjectivity. Eur Arch Psychiatry Clin Neurosci (2013) 263(4):353-64. doi: 10.1007/s00406-012-0366-z

8. Kendler KS. Toward a limited realism for psychiatric nosology based on the coherence theory of truth. Psychol Med (2015) 45(6):1115-8. doi: 10.1017/ S0033291714002177

9. Hasler G, Drevets WC, Manji HK, Charney DS. Discovering endophenotypes for major depression. Neuropsychopharmacology (2004) 29(10):1765-81. doi: 10.1038/sj.npp.1300506

10. Stoyanov DS. The endophenotype project and the validation theory: integration of neurobiology and psychiatry. Folia Med (Plovdiv) (2010) 52(1):18-25.

11. Loewenthal KM. An introduction to psychological tests and scales. London: Psychology Press (2001). p. 172.

12. Stoyanov D, Kandilarova S, Borgwardt S, Stieglitz R-D, Hugdahl K, Kostianev S. Psychopathology assessment methods revisited: on translational cross-validation of clinical self-evaluation scale and fMRI. Front Psychiatry (2018) 9:21. doi: 10.3389/fpsyt.2018.00021

13. von Zerssen D. Clinical Self-Rating Scales (CSRS) of the Munich Psychiatric Information System (PSYCHIS München). In: Sartorius N, Ban TA, editors. Assessment of depression. Berlin: Springer (1986). doi: 10.1007/978-3-642-70486-4_25

\section{ETHICS STATEMENT}

The studies involving human participants were reviewed and approved by Research Ethics Committee, Plovdiv Medical University. The patients/participants provided their written informed consent to participate in this study.

\section{AUTHOR CONTRIBUTIONS}

DS has developed the conceptual rationale and delivered the text in the Introduction and a major part of the Discussion. SK has performed the statistical analysis and delivered the Methods and Results sections. ZA contributed to the interpretation of the Results and the Discussion sections. AS consulted the data analysis procedures and edited the manuscript. SB consulted the development of the paradigm and the data processing, and edited the paper and supervised the entire project. RP was involved in the empirical study and data processing.

14. Stoyanov D, Kandilarova S, Sirakov N, Stoeva M, Velkova K, Kostianev S. Towards translational cross-validation of clinical psychological tests and fMRI: experimental implementation. C R Acad Bulg Sci (2017) 70(6):6.

15. Sheehan DV, Lecrubier Y, Sheehan KH, Amorim P, Janavs J, Weiller E, et al. The Mini-International Neuropsychiatric Interview (M.I.N.I.): the development and validation of a structured diagnostic psychiatric interview for DSM-IV and ICD-10. J Clin Psychiatry (1998) 59 Suppl 20:22-33:quiz 4-57.

16. Montgomery SA, Asberg M. A new depression scale designed to be sensitive to change. Br J Psychiatry (1979) 134:382-9. doi: 10.1192/bjp.134.4.382

17. Kay SR, Fiszbein A, Opler LA. The positive and negative syndrome scale (PANSS) for schizophrenia. Schizophr Bull (1987) 13(2):261-76. doi: 10.1093/schbul/13.2.261

18. Seghier ML. The angular gyrus: multiple functions and multiple subdivisions. Neuroscientist (2013) 19(1):43-61. doi: 10.1177/1073858412440596

19. Niznikiewicz M, Donnino R, McCarley RW, Nestor PG, Iosifescu DV, O’Donnell B, et al. Abnormal angular gyrus asymmetry in schizophrenia. Am J Psychiatry (2000) 157(3):428-37. doi: 10.1176/appi.ajp.157.3.428

20. Farrer C, Franck N, Frith CD, Decety J, Georgieff N, d'Amato T, et al. Neural correlates of action attribution in schizophrenia. Psychiatry Res (2004) 131(1):31-44. doi: 10.1016/j.pscychresns.2004.02.004

21. Maddock RJ, Garrett AS, Buonocore MH. Remembering familiar people: the posterior cingulate cortex and autobiographical memory retrieval. Neuroscience (2001) 104(3):667-76. doi: 10.1016/S0306-4522(01)00108-7

22. Zhou Y, Shu N, Liu Y, Song M, Hao Y, Liu H, et al. Altered resting-state functional connectivity and anatomical connectivity of hippocampus in schizophrenia. Schizophr Res (2008) 100(1-3):120-32. doi: 10.1016/j. schres.2007.11.039

23. Cavanna AE, Trimble MR. The precuneus: a review of its functional anatomy and behavioural correlates. Brain (2006) 129(Pt 3):564-83. doi: 10.1093/ brain/awl004

24. Warrier C, Wong P, Penhune V, Zatorre R, Parrish T, Abrams D, et al. Relating structure to function: Heschl's gyrus and acoustic processing. J Neurosci (2009) 29(1):61-9. doi: 10.1523/JNEUROSCI.3489-08.2009

25. Nelson SM, Dosenbach NUF, Cohen AL, Wheeler ME, Schlaggar BL, Petersen SE. Role of the anterior insula in task-level control and focal attention. Brain Struct Funct (2010) 214(5-6):669-80. doi: 10.1007/s00429-010-0260-2

26. Davey CG, Harrison BJ, Yucel M, Allen NB. Regionally specific alterations in functional connectivity of the anterior cingulate cortex in major depressive disorder. Psychol Med (2012) 42(10):2071-81. doi: 10.1017/ S0033291712000323

27. Keedwell PA, Andrew C, Williams SC, Brammer MJ, Phillips ML. The neural correlates of anhedonia in major depressive disorder. Biol Psychiatry (2005) 58(11):843-53. doi: 10.1016/j.biopsych.2005.05.019 
28. Mehrabian A. Beyond IQ: broad-based measurement of individual success potential or "emotional intelligence". Genet Soc Gen Psychol Monogr (2000) 126(2):133-239.

29. Rockstuhl T, Seiler S, Ang S, Dyne LV, Annen H. Beyond general intelligence (IQ) and emotional intelligence (EQ): the role of cultural intelligence (CQ) on cross-border leadership effectiveness in a globalized world. J Soc Issues (2011) 67(4):15. doi: 10.1111/j.1540-4560.2011.01730.x

30. Skodlar B. Three different meanings of depression in schizophrenia. A phenomenological perspective. Psychiatr Danub (2009) 21 Suppl 1:88-92.

31. Gramaglia C, Pietrini F, Zeppegno P. Symptoms of depression: "hot" and "cold" cognition. Evid-Based Psychiatr Care (2015) 1:48-58.

32. Gualtieri CT, Morgan DW. The frequency of cognitive impairment in patients with anxiety, depression, and bipolar disorder: an unaccounted source of variance in clinical trials. J Clin Psychiatry (2008) 69(7):1122-30. doi: 10.4088/JCP.v69n0712
33. Lee RS, Hermens DF, Porter MA. Redoblado-Hodge MA. A meta-analysis of cognitive deficits in first-episode Major Depressive Disorder. J Affect Disord (2012) 140(2):113-24 doi: 10.1016/j.jad.2011.10.023.

Conflict of Interest: The authors declare that the research was conducted in the absence of any commercial or financial relationships that could be construed as a potential conflict of interest.

Copyright (c) 2019 Stoyanov, Kandilarova, Arabadzhiev, Paunova, Schmidt and Borgwardt. This is an open-access article distributed under the terms of the Creative Commons Attribution License (CC BY). The use, distribution or reproduction in other forums is permitted, provided the original author(s) and the copyright owner(s) are credited and that the original publication in this journal is cited, in accordance with accepted academic practice. No use, distribution or reproduction is permitted which does not comply with these terms. 